\title{
Drinking Water as Iron Carrier for the Prevention of Iron Deficiency Anemia: The Brazilian Experience
}

\author{
Jose E. Dutra-de-Oliveira ${ }^{1}$, Júlio S. Marchini ${ }^{1}$, Joel Lamounier ${ }^{2}$, Carlos A. N. de Almeida ${ }^{3}$ \\ ${ }^{1}$ Department of Internal Medicine, Ribeirao Preto School of Medicine, University of Sao Paulo, Ribeirao Preto, Brazil \\ ${ }^{2}$ Department of Pediatrics, Medical School Belo Horizonte, Belo Horizonte, Brazil \\ ${ }^{3}$ Department of Pediatrics, Ribeirão Preto School of Medicine, University of Ribeirao Preto, Ribeirao Preto, Brazil \\ Email: jeddoliv@fmrp.usp.br
}

Received June 28, 2013; revised July 30, 2013; accepted August 7, 2013

Copyright (C) 2013 Jose E. Dutra-de-Oliveira et al. This is an open access article distributed under the Creative Commons Attribution License, which permits unrestricted use, distribution, and reproduction in any medium, provided the original work is properly cited.

\begin{abstract}
On a global base, estimated 2 billion people are iron deficient and/or anemic with small children and child bearing age most likely to be the ones affected. These high levels of world anemia prevalence have been maintained and even growing in spite of the global and national food fortification programmes carried out in several places. These have been based on the iron fortification of local foods. In Brazil a wheat flour iron fortification program has been going on for several years and the anemia prevalence has been going on as a still large public health problem. We started since the nineties and in several under five day care centers, in various part of Brazil a new program using drinking water as the iron carrier for the prevention of iron deficiency anemia. It was shown through several studies in different places in Brazil that drinking water being available everywhere, daily consumed by everyone, children, adults and old people have shown to be effective for the prevention of iron anemia. Iron salts are low-priced, water soluble, effective, easily and locally prepared, should be known and accepted as a rational, practical and effective locally community preventive solution for iron anemia, still a great problem of our developing and underdeveloped countries of the world.
\end{abstract}

Keywords: Iron Anemia; Iron Carrier; Control Anemia; Drinking Water Fortification; Community Studies and Support

\section{Introduction}

Water is an essential nutrient for all known forms of life and the mechanism by which fluid and electrolyte homeostasis is maintained in humans A $65 \mathrm{~kg}$ man contains about 40 liters of water. Of this, 25 liters are intracellular and 15 liters are extracellular. These fluids are in continuously balance, being turned constant by a water intake and output. Several substances are found in drinking water that significantly contributes to health and wellbeing. Water acts as a building material, in thermoregulation and body metabolism. It has been shown it could be a carrier of numerous macro and micronutrients as $\mathrm{Ca}$, $\mathrm{F}, \mathrm{Cu}, \mathrm{Mg}$, Se, $\mathrm{K}$ and particularly low in iron and iodine, source of health problems in various parts of our organism $[1,2]$.

Globally, an estimated two billion lives are affected by a deficiency of essential micronutrients, known as hidden hunger $[2,3]$. Worldwide, the most widespread micronutrient deficiencies are iron, iodine, zinc, vitamin A and folate, four of them soluble in water. Iron deficiency anemia is the most common disorder in the world [2]. Even mild to moderate deficiencies of micronutrients lead to impaired intellectual and growth development, increased morbidity from infectious diseases in infants and young children and decreased work productivity in adults [3].

Iron deficiency and iron anemia are known today as the main, the most frequent worldwide nutritional deficiency problem in the world. Millions of children are still anemic or becoming anemic today in the world. It is generally considered to be the result of low iron intake from local diets. It is the most common health problem of children and pregnant women of underdeveloped and developing countries, in spite of the large amount of attention and effort carried out by national and international organizations.

In Brazil iron deficiency is the most significant nutritional health problem, present all over the country [4]. It is found in the less developed areas of our country, even 
in some developed ones, affecting mostly children three to four, five years old, mainly of poor families. Studies published out in 2003 in Brazil showed an increased number of children with iron anemia from $20 \%$ in 1975 1980 to $47 \%$ in $1995 / 2000$ [5] and over 50\% - 60\% prevalence has been reported in some areas of Brazil in 2011 [4]. Control and prevention of iron anemia are mainly carried out in Brazil through Government programs of iron fortification of wheat flour products. Pharmacological iron supplements for the treatment of anemia are supposed to be available and supplied by the Brazilian Government medical attention programs.

We have been studying and carrying on since the nineties [6] a new approach for prevention of iron deficiency anemia through iron fortification with iron sulfate added to drinking water at daycare community centers, were preschool children attend daily. Most of the time we use ferrous sulfate $\left(\mathrm{FeSO}_{4} \cdot 7 \mathrm{H}_{2} \mathrm{O}\right)$, but other iron soluble salts were also studied. A concentrate ferrous sulfate solution is prepared at a local health center and a local daycare community attendant learn the amount of the concentrate iron solution that should be added to 20 liter to have 10 $20 \mathrm{mg}$ of iron per liter. The iron fortified drinking water is available and consumed daily ad libitum by each child. Iron salts particularly iron sulfate are pharmacy available, not expensive, hydrosoluble and do not require any special technology, just addition of it to a local drinking water bottle supply. Change of color and taste of the ferrous sulfate fortified drinking water were small and had been shown not to be a problem for the children intake. Our initial animal experiments demonstrate that anemia could be prevented by iron fortified drinking water. It was carried out in young rats that drank iron-fortified water with several soluble iron salts as iron sulfate, sodium iron EDTA, ferric orthophosphate and iron glycine chelate (GLY) $[7,8]$. Similar children studies were carried out with these different iron sources as with iron sodium EDTA, showed to be a high-quality water bioavailable source of iron and produced less color and taste changes in the drinking water. It is more expensive than ferrous sulfate. Ferrous sulfate was shown to be the best one, considering high availability and low price.

Several other studies were carried out by our group in preschool children attending day-care centers at medium and small communities and in different parts of Brazil [6, 9-12]. Each one of our studies lasted for four, six to eight months. Each participant child had, from the beginning to end of the studies, a personal clinical and medical nutritional check up, including blood hemoglobin levels and in some of the studies levels of blood ferritin were also checked and followed. In the world, iron status of women was associated with the iron concentration of potable water in rural Bangladesh [13].

\section{Brazilian Drinking Water Iron Carrier Studies}

\subsection{Drinking Water as an Iron Carrier to Control Anemia in Preschool Children in a Day-Care Center}

J. E. Dutra-de-Oliveira, J. B. Ferreira, V. P. Vasconcellos, J. S. Marchini. Journal American College Nutrition, 1994 [6].

Several foods have been used as iron $(\mathrm{Fe})$ carriers to fight national and global iron deficiency and anemia. This paper describes the longitudinal effect of Fe-fortified drinking water given to a group of Brazilian preschool children. The experimental design included 31 preschool children who attended a day-care institution. Hemoglobin and serum ferritin were the blood parameters used to check the Fe status. Ferrous sulfate $(20 \mathrm{mg}$ $\mathrm{Fe} / \mathrm{L}$ ) was added daily to their drinking water container and laboratory measurements were obtained before the addition, 4 and 8 months later. The number of Fe-deficient children decreased drastically after they started drinking the Fe-enriched water. Mean hemoglobin values increased from 10.6 to $13.7 \mathrm{~g} / \mathrm{dL}$ and serum ferritin from 13.7 to $25.6 \mu \mathrm{g} / \mathrm{L}$. There were no problems related to the presence of ferrous salt addition or to the children drinking the Fe-enriched water. Fe-enriched drinking water was shown to be a practical alternative way to supply $\mathrm{Fe}$ to children attending day-care institutions. Iron deficiency affects more than one billion people worldwide, although it is most common among young children and women of childbearing age. Poor iron status has severe nutritional and health consequences. The authors describe the longitudinal effect of iron-fortified drinking water given to a group of Brazilian preschool children as a way of fighting iron deficiency and anemia. The status of 31 preschool children attending a day-care institution for low socioeconomic families in Ribeirao Preto, Brazil was followed from November 1990 to October 1991. Iron sulfate was added daily to the subjects' drinking water container. Measurements of hemoglobin and serum ferritin levels in the children were taken before the addition and four and eight months later, to evaluate iron status. Mean hemoglobin values increased from 10.6 to $13.7 \mathrm{~g} / \mathrm{dL}$ and serum ferritin from 13.7 to $25.6 \mu \mathrm{g} / \mathrm{L}$ with no problems reported to the iron salt addition or to the children drinking the iron-enriched water. The number of iron-deficient children decreased drastically after they began drinking the iron-enriched water. It may therefore be concluded that iron-enriched drinking water is a practical alternative to supply iron to children attending a day-care institution [6]. 


\subsection{Effect of Fortification of Drinking Water with Iron Plus Ascorbic Acid or with Ascorbic Acid Alone on Hemoglobin Values and Anthropometric Indicators in Preschool Children in Day-Care Centers in Southeast Brazil}

C. A. N. de Almeida, J. E. Dutra-de-Oliveira, G. C. L. Crott, A. Cantolini, R. G. Ricco, L. A. Del Ciampo, M. E. Baptista. Food and Nutrition Bulletin, 2005 [9].

Iron-deficiency anemia currently is the most frequently occurring nutritional disorder world-wide. Previous Brazilian studies have demonstrated that drinking water fortified with iron and ascorbic acid is an adequate vehicle for improving the iron supply for children attending day-care centers. The objective of this study was to clarify the role of ascorbic acid as a vehicle for improving iron uptake in children in day-care centers in Brazil. A six-month study was conducted on 150 children attending six day-care centers divided into two groups of three day-care centers by drawing lots: the Iron-C group (3 day-care centers, $n=74$ ), which used water fortified with $10 \mathrm{mg}$ elemental iron $\left(\mathrm{FeSO}_{4} \cdot 7 \mathrm{H}_{2} \mathrm{O}\right)$ and $100 \mathrm{mg}$ ascorbic acid per liter, and the comparison group (3 day-care centers, $n=76$ ), which used water containing $100 \mathrm{mg}$ ascorbic acid per liter. Anthropometric measurements and determinations of capillary hemoglobin were performed at the beginning of the study and after six months of intervention. The food offered at the day-care centers was also analyzed. The food offered at the daycare center was found to be deficient in ascorbic acid, poor in heme iron, and adequate in non-heme iron. Supplementation with fortified drinking water resulted in a decrease in the prevalence of anemia and an increase in mean hemoglobin levels associated with height gain in both groups. Fortification of drinking water with iron has previously demonstrated effectiveness in increasing iron supplies. This simple strategy was confirmed in the present study. The present study also demonstrated that for populations receiving an abundant supply of non-heme iron, it is possible to control anemia in a simple, safe, and inexpensive manner by adding ascorbic acid to drinking water [9].

\subsection{Fortification of Drinking Water to Control Iron-Deficiency Anemia in Preschool Children.}

J. E. Dutra-de-Oliveira, J. Lamounier, C. A. N. de Almeida, J. S. Marchini. Food and Nutrition Bulletin, 2007 [11].

Iron-deficiency anemia is the most common type of micronutrient malnutrition in the world. Its etiology and control have been studied, but the problem is kept present and is increasing in some countries. Iron fortification of mass-consumption foods is considered one of the most viable approaches to deliver bioavailable iron to the population. Drinking water with added iron compounds $\left(\mathrm{FeSO}_{4} \cdot 7 \mathrm{H}_{2} \mathrm{O}\right)$ was offered to preschool children at daycare centers in Brazil. Iron solutions were prepared with $10 \mathrm{mg}$ to $20 \mathrm{mg}$ iron/L. Clinical and anthropometric measurements and blood hemoglobin concentrations were obtained at the beginning of each study and 4 to 8 months later. No problems with acceptability or side effects were observed. Daily water intake by children was around 500 ml. Iron-deficiency anemia was found in all studies. Control children not receiving iron supplementation maintained their initial hemoglobin level. Anemia was reduced in the groups receiving iron-fortified drinking water. Based on physical properties, tests in animals and studies on preschool children at Brazilian Day-care Centers showed that drinking water locally fortified with iron compounds should be considered and used as a worldwide available vehicle to control iron-deficiency anemia. Drinking water, besides being universally accessible, is a locally available vehicle, easily fortified, can be a vehicle for hydrosoluble iron and reduces iron-deficiency anemia in preschool children [11].

\subsection{Domestic Drinking Water-An Effective Way to Prevent Anemia among Low Socioeconomic Families in Brazil}

J. E. Dutra-de-Oliveira, C. A. N. de Almeida. Food and Nutrition Bulletin, 2002 [14].

Iron deficiency and iron-deficiency anemia are common in developing world. We evaluated the feasibility of iron fortification of domestic drinking water to prevent and control iron deficiency and iron-deficiency anemia. Twenty-one families representing 88 persons, including children, were selected to participate in this study. Twelve families added an iron solution plus ascorbic acid (10 mg elemental iron and $60 \mathrm{mg}$ of ascorbic acid per liter) to their domestic drinking water over a four months period and nine families added a placebo. Blood samples were collected, before and after the four months, for hemoglobin and serum ferritin measurements. Iron-fortified drinking water increased hemoglobin (children $10.9+1.1$ $\mathrm{g} / \mathrm{dL}$ to $11.7+1.1 \mathrm{~g} / \mathrm{dL} p<0.01$, adults $12.9+1.7 \mathrm{~g} / \mathrm{dL}$ to $13.7+1.7 \mathrm{~g} / \mathrm{dL} p<0.01$ ) and ferritin (children $27.6+$ $21.6 \mathrm{ng} / \mathrm{dL}$ to $33.8+22.1 \mathrm{ng} / \mathrm{dL}$, adults $74.8+41.3$ ng/dL to $106.2+93.9 \mathrm{ng} / \mathrm{dL} p<0.05)$. No significant changes in hemoglobin and ferritin were found in the placebo group after 4 months. Preparation, distribution, and consumption of the solutions were successful. Iron fortification of household drinking water can be a simple and effective alternative to deal with iron deficiency and iron-deficiency anemia in less developed areas [14]. 


\subsection{Iron Fortification of Domestic Drinking Water to Prevent Anemia among Low Socioeconomic Families in Brazil}

J. E. Dutra-de-Oliveira, M. M. Scheid, I. D. Desai, J. S. Marchini. International Journal of Food Science and Nutrition, 1996 [15]

Iron deficiency anemia is the most common micronutrient deficiency affecting mostly the low socioeconomic populations of the developing world. The objective of this study was to evaluate the feasibility of iron fortification of household drinking water to prevent iron deficiency anemia among members of the low socioeconomic families of Southern Brazil. A total of 21 low socioeconomic families representing 88 subjects including 1 - 6 years old children whose hemoglobin level was between 10 and $12 \mathrm{~g} / \mathrm{dL}$ were selected to participate in this study. Nine families in the control group were supplied with placebo solution and 12 families in the experimental group were supplied iron solution with ascorbic acid (10 mg elemental iron per liter) to be added to their domestic drinking water over a period of 4 months. The feasibility and acceptability of iron fortified drinking water was assessed through home visits and questionnaires. Blood samples were collected at the beginning and after 4 months of the study for the determination of hemoglobin and serum ferritin levels. The results of this study indicated that iron fortified drinking water was well received by the low socioeconomic families and that it was effective in improving the hemoglobin and serum ferritin levels. It can be concluded from this study that iron fortification of household drinking water is a simple and effective alternative for developing countries along with other technological approaches to iron fortification of foods [15].

\subsection{Randomized Controlled Trial of Iron-Fortified Drinking Water in Preschool Children}

F. P. N. Arcanjo, O. M. S. Amâncio, J. A. P. Braga, V. P. T. Pinto. Journal of the American College of Nutrition, 2010 [16].

To evaluate the effects of fortified drinking water, with different concentrations of iron added, on hemoglobin and hematocrit values in preschoolers. Double-blind, randomized cluster clinical trial, with children aged 2 to 5 years of age, from 4 state-run schools, forming 1 group for each school. For fortification, ferrous sulfate $\left(\mathrm{FeSO}_{4} \cdot 7 \mathrm{H}_{2} 0\right)$ in concentrations of $5 \mathrm{mg}$ of elemental iron per liter of water (group A), $7.5 \mathrm{mg}$ (group B), and $10 \mathrm{mg}$ (group C), was used during a period of 4 months. In group $\mathrm{D}$, the control, a placebo (Bixa Oreland) was added. Hemoglobin and hematocrit values were checked before and after intervention. Before fortification, hemoglobin and hema- tocrit averages were below the reference values adopted in all groups. After fortification, the prevalence of anemia showed a reduction in the 4 groups, which was more pronounced in group B, at $48.3 \%$. The hemoglobin values in groups B (11.5) and C (11.4) were statistically similar. However, the average consumption of water/day/student was lower in group C. Comparison of hemoglobin values between groups A (11.2) and D (11.0) did not show a significant difference, suggesting insignificant efficacy with $5 \mathrm{mg} \mathrm{Fe} / \mathrm{L}$ fortification. The consumption of drinking water fortified with $7.5 \mathrm{mg}$ of elemental iron/L water resulted in greater adhesion and an increase in hemoglobin values, with a reduction in the prevalence of anemia [16].

\section{Discussion}

Iron deficiency and iron anemia are the most common and widespread nutritional disorder of the world. It is a major public health problem developing and underdeveloped countries. It has been primarily linked to a poor diet with low bioavailable iron content. The role and the work carried out all over the world with a large group of food fortification programs for the control of micronutrient deficiencies including iron deficiency was reviewed and concluded that food fortification with micronutrients would be the best way to deal with world micronutrient problems [2,3]. It has been said that food fortification can make an important contribution for the prevention of iron deficiency and iron anemia. Wheat flour iron fortification law has not been successful for the prevention of iron anemia in Brazil. Drinking water iron fortification has been shown in Brazil an effective strategy for the prevention of iron deficiency and anemia in small children. Iron water fortification does not require change in food habits, does not alter the drinking water habits of the population. Can be introduced quickly, is safe and can be a cost-effective way of reaching daily large target populations, children and adults, which are at risk of iron deficiency and anemia.

\section{Conclusions}

Iron deficiency and iron anemia are the most common and widespread public health problem in the world, mainly affecting the developing and underdeveloped countries. It has been linked to the low iron intake from their diets. The best way to prevent iron anemia is to increase the intake of iron, the iron fortification of their usual foods. Wheat flour, pasta, milk, salt, sugar, soya, soft drinks and other foods have been iron fortified but iron deficiency/ anemia is still present and even growing in several places of the world.

Our original studies in Brazil on the use of iron fortification of drinking water for the prevention of iron ane- 
mia showed that drinking water, being available everywhere, daily consumed by everyone, children, adults and old people have been shown to be effective for the prevention of iron anemia. Iron salts are low-priced, water soluble, effective, easily and locally prepared, should be known and accepted as a rational, practical and professional preventive solution for the iron anemia, still a great problem of our developing and underdeveloped countries of the world.

\section{Acknowledgements}

We acknowledge the support of FAEPA, Foundation for Teaching Research and Community Services of Ribeirao Preto, SP, Brazil.

\section{REFERENCES}

[1] Word Health Organization, "Nutrients in Drinking Water," Water, Sanitation and Health Protection and the Human Environment World Health Organization, WHO, Geneva, 2005.

[2] Word Health Organization, "Iron Deficiency Anaemia. Assessment, Prevention, and Control. A Guide for Programme Managers,” United Nations Children's Fund, United Nations University, Word Health Organization, Geneva, 2001.

[3] L. Allen, B. Benoist, O. Dary and R. Hurrell, "Guidelines on Food Fortification with Micronutrients," WHOWord Health Organization, FAO-Food and Agricultural Organization of the United Nations, WHO, Geneva, 2006.

[4] D. S. Martins, "Anemia Prevalence in Children and Adolescents of the Last Eleven Years in Brazil,” MS Dissertation, Federal University of Rio Grande do Sul, Porto Alegre, 2011.

[5] M. B. Filho and A. Rissin, "Nutritional Transition in Brazil: Geographic and Temporal Trends," Caderno de Saude Publica, Vol. 19, Suppl. 1, 2003, pp. S181-S191.

[6] J. E. Dutra-de-Oliveira, J. B. Ferreira, V. P. Vasconcellos and J. S. Marchini, "Drinking Water as an Iron Carrier to Control Anemia in Preschool Children in a Day-Care Center," Journal of the American College of Nutrition, Vol. 13, No. 2, 1994, pp. 198-202.

[7] J. E. Dutra-de-Oliveira, M. L. Freitas, J. F. Ferreira, A. L. Gonçalves and J. S. Marchini, "Iron from Complex Salts and Its Bioavailability to Rats," International Journal for Vitamin and Nutrition Research, Vol. 65, No. 4, 1995, pp. 272-275.
[8] L. Hallberg, L. Rossander-Hulthén and E. Gramatkovski, "Iron Fortification of Flour with a Complex Ferric Orthophosphate," The American Journal of Clinical Nutrition, Vol. 50, No. 1, 1989, pp. 129-135.

[9] C. A. N. de Almeida, J. E. Dutra-de-Oliveira, G. C. Crott, A. Cantolini, R. G. Ricco, L. A. Del Ciampo and M. E. Baptista, "Effect of Fortification of Drinking Water with Iron Plus Ascorbic Acid or with Ascorbic Acid Alone on Hemoglobin Values and Anthropometric Indicators in Preschool Children in Day-Care Centers in Southeast Brazil,” Food \& Nutrition Bulletin, Vol. 26, No. 3, 2005, pp. 259-265.

[10] C. A. N. de Almeida, G. C. L. Crott, R. G. Ricco, L. A. Del Ciampo, J. E. Dutra-de-Oliveira and A. Cantolini, "Control of Iron-Deficiency Anaemia in Brazilian Preschool Children Using Iron-Fortified Orange Juice,” $\mathrm{Nu}$ trition Research, Vol. 23, No. 1, 2003, pp.27-33. doi:10.1016/S0271-5317(02)00487-6

[11] J. E. Dutra-de-Oliveira, J. Lamounier, C. A. N. de Almeida and J. S. Marchini, "Fortification of Drinking Water to Control Iron-Deficiency Anemia in Preschool Children," Food \& Nutrition Bulletin, Vol. 28, No. 3, 2007, pp. 173180.

[12] J. E. Dutra-de-Oliveira, S. Ventura, A. M. Souza and J. S. Marchini, "Iron Deficiency Anemia in Children: Prevalence and Prevention Studies in Ribeirão Preto, Brazil," Archivos Latinoamericanos Nutricion, Vol. 47, No. 2, 1997, pp. 41-43.

[13] R. D. Merrill, A. A. Schamin, H. Ali, N. Jahan, A. B. Labrique, K. Schulze, P. Christian and K. P. West Jr., "Iron Status of Women Is Associated with the Iron Concentration of Potable Groundwater in Rural Bangladesh," The Journal of Nutrition, Vol. 141, No. 5, 2011, pp. 944949. doi:10.3945/jn.111.138628

[14] J. E. Dutra-de-Oliveira, C. A. N. de Almeida, "Domestic Drinking Water-An Effective Way to Prevent Anemia among Low Socioeconomic Families in Brazil,” Food \& Nutrition Bulletin, Vol. 23, No. 3, 2002, pp. 213-216.

[15] J. E. Dutra-de-Oliveira, M. M. Scheid, I. D. Desai and J. S. Marchini, "Iron Fortification of Domestic Drinking Water to Prevent Anemia among Low Socioeconomic Families in Brazil," International Journal of Food Sciences and Nutrition, Vol. 4, No. 3, 1996, pp. 213-219. doi:10.3109/09637489609012583

[16] F. P. N. Arcanjo, O. M. S. Amancio, J. A. P. Braga and V. P. T. Pinto, "Randomized Controlled Trial of Iron-Fortified Drinking Water in Preschool Children," Journal of the American College of Nutrition, Vol. 29, No. 2, 2010, pp. 122-129. doi:10.1080/07315724.2010.10719825 